\title{
P52 The Long-term Effects of Transcatheter Aortic Valve Implantation on Aortic Stiffness
}

Vasiliki Gardikioti1 ${ }^{1, *}$, Dimitrios Terentes-Printzios ${ }^{1}$, Charalambos Vlachopoulos ${ }^{1}$, Konstantinos Toutouzas ${ }^{1}$, Evangelia Christoforatou ${ }^{1}$, Maria Xanthopoulou ${ }^{1}$, Georgios Benetos ${ }^{1}$, Georgios Latsios ${ }^{1}$, Gerasimos Siasos ${ }^{1}$, Evangelia Mpei $^{1}$, Manolis Vavuranakis ${ }^{1}$, Dimitrios Tousoulis ${ }^{1}$

${ }^{1}$ First Department of Cardiology, Hippokration General Hospital, Athens, Greece

\section{ABSTRACT}

Purpose/Background/Objectives: Aortic stiffness and hemodynamics are independent predictors of adverse cardiovascular events. Transcatheter aortic valve implantation (TAVI) is growingly used in high surgical risk patients with aortic valve stenosis. We sought to investigate the effect of TAVI on aortic stiffness.

Methods: Fifty-five high-risk patients (mean age $79.5 \pm 8.8$ years, $46 \%$ males) with severe symptomatic aortic stenosis undergoing TAVI were included. Arterial stiffness was estimated through carotid-femoral pulse wave velocity (cfPWV). Measurements were conducted prior to the implantation, at discharge and 1 year post-TAVI.

Results: Prior to the implantation, cfPWV was $7.5 \pm 1.4 \mathrm{~m} / \mathrm{s}$ for the overall population. There was a statistically significant increase in measurements of aortic stiffness at discharge $(7.5 \pm 1.4$ vs $8.3 \pm 1.9 \mathrm{~m} / \mathrm{s}$ for cfPWV with $p=0.043)$ without a statistically significant change in SBP $(p=$ NS) (Figure 1). There was also a significant increase in cfPWV $(8.7 \pm 1.8 \mathrm{~m} / \mathrm{s})$ after 1 year compared to the pre-procedural assessment $(p=0.001)$. We also observed a non-significant increase in peripheral SBP at 1 year compared to SBP measurements at baseline and at discharge $(153 \pm 24 \mathrm{~m} / \mathrm{s}$ vs $147 \pm 21$ and $148 \pm 22 \mathrm{~m} / \mathrm{s}$, respectively; $p=0.319$ ) and these changes were independent of age and gender (Figure 1).

Conclusion: Our study shows that both shortly as well as in the long-term after TAVI subjects experience an increase in aortic stiffness that is independent of changes in SBP. These findings further elucidate the hemodynamic consequences and provide evidence of a possible long-standing repercussion of TAVI.

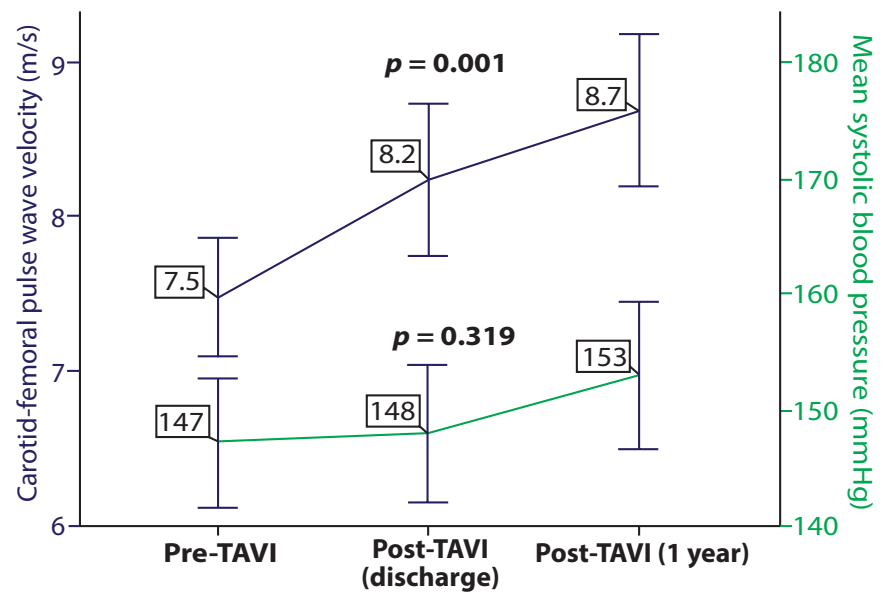

Figure 1

(c) 2019 Association for Research into Arterial Structure and Physiology. Publishing services by Atlantis Press International B.V. This is an open access article distributed under the CC BY-NC 4.0 license (http://creativecommons.org/licenses/by-nc/4.0/). 